\title{
Precision Pulsar Timing with NASA's Deep Space Network
}

\author{
Lawrence Teitelbaum ${ }^{1}$, Walid Majid ${ }^{1}$, Manuel M. Franco ${ }^{1}$, Daniel \\ J. Hoppe ${ }^{1}$, Shinji Horiuchi ${ }^{2}$ and T. Joseph W. Lazio ${ }^{1}$ \\ ${ }^{1}$ Jet Propulsion Laboratory, California Institute of Technology, 4800 Oak Grove Dr., \\ M/S 67-201, Pasadena, CA 91109 USA \\ email: Walid.A.Majid@jpl.nasa.gov \\ ${ }^{2}$ Canberra Deep Space Communications Complex, 421 Discovery Dr., Paddys River ACT \\ 2620, Australia
}

\begin{abstract}
Millisecond pulsars (MSPs) are a class of radio pulsars with extremely stable rotation. Their excellent timing stability can be used to study a wide variety of astrophysical phenomena. In particular, a large sample of these pulsars can be used to detect low-frequency gravitational waves. We have developed a precision pulsar timing backend for the NASA Deep Space Network (DSN), which will allow the use of short gaps in tracking schedules to time pulses from an ensemble of MSPs. The DSN operates clusters of large dish antennas (up to 70-m in diameter), located roughly equidistant around the Earth, for communication and tracking of deep-space spacecraft. The backend system will be capable of removing entirely the dispersive effects of propagation of radio waves through the interstellar medium in real-time. We will describe our development work, initial results, and prospects for future observations over the next few years.
\end{abstract}

Keywords. gravitational waves, instrumentation: detectors, pulsars: general

\section{Introduction}

The Deep Space Network (DSN) is the set of radio antennas used by NASA to command, communicate with, and track deep space spacecraft. $\dagger$ In order to provide near continuous coverage with spacecraft anywhere in the solar system, the DSN operates 13 radio antennas at three complexes approximately equidistant around Earth (Goldstone, California; Canberra, Australia; and Madrid, Spain). Each complex has one $70 \mathrm{~m}$ diameter antenna and three or more $34 \mathrm{~m}$ diameter antennas. While the primary function of the DSN is spacecraft tracking, there is time available for scientific observations (Lazio et al. 2015).

Using antennas within the DSN, Hellings \& Downs (1983) attempted the first implementation of a pulsar timing array to detect a stochastic gravitational wave background. They were unsuccessful, with one primary reason being that they carried out their initial attempt before the discovery of the first millisecond pulsar in 1982 (Backer et al. 1982).

The deep space spectral allocations are near $2.2 \mathrm{GHz}, 8 \mathrm{GHz}$, and $32 \mathrm{GHz}$. However, these spectral allocations are relatively narrowband by modern radio astronomical standards, and, combined with the relatively steep spectral indices of pulsars (Bates et al. 2013), precision timing of most millisecond pulsars is not possible within these bands. In 1988, the $70 \mathrm{~m}$ antennas were also outfitted with a system operating near $1.7 \mathrm{GHz}$ (Withington 1988), designed to track the international Vega missions to Venus, which also included flybys of Halley's comet. Again, based on the spacecraft tracking needs, this feed system was also relatively narrowband $(90 \mathrm{MHz})$.

$\dagger$ A real-time view of DSN spacecraft tracking is at http://eyes.nasa.gov/dsn/dsn.html. 

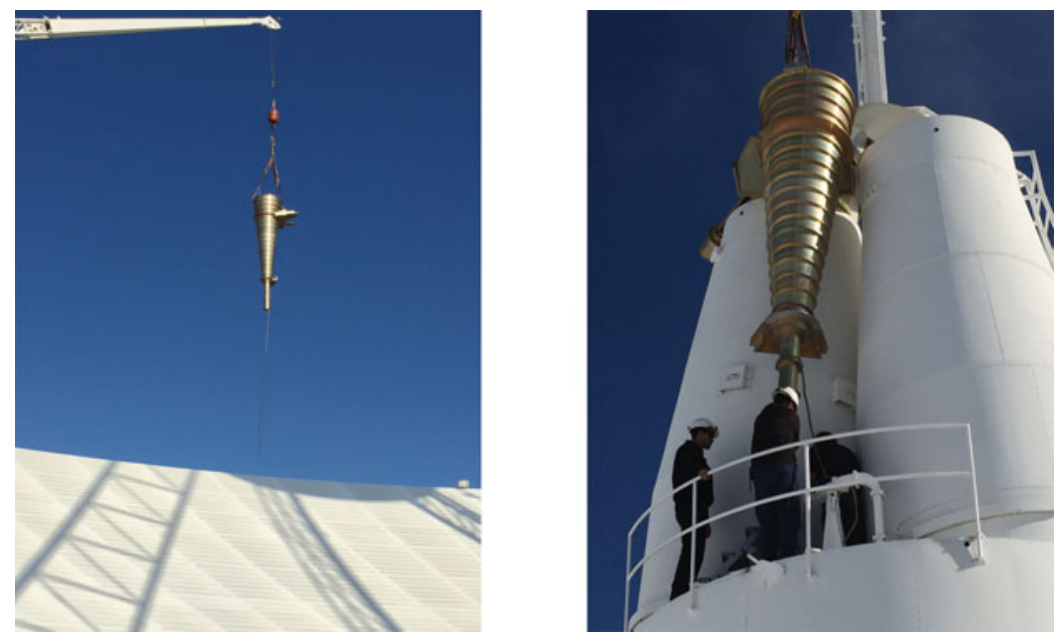

Figure 1. Installation of the new wideband feed on 2015 August 21. (Left) New 1.4-1.9 GHz feed being lifted onto the $70 \mathrm{~m}$ DSS-43 antenna at the Canberra Deep Space Communications Complex. (Right) Installation of the new feed into the Cassegrain focus "cone" of DSS-43. The scale of the new feed is evident by comparison with the individuals in the image.

To enable multi-wavelength monitoring of energetic sources detected by NASA's Fermi Telescope, the receivers downstream of the feed were retrofitted with wide-band, cooled filters to increase sensitivity by enabling approximately $500 \mathrm{MHz}$ bandwidth $(1.4-1.9 \mathrm{GHz})$. However, the system remained relatively narrowband due to the presence of the feed.

\section{Wideband Feed System}

In the southern hemisphere, there is only a single telescope currently capable of conducting precision pulsar timing measurements, the $64 \mathrm{~m}$ Parkes Radio Telescope. Motivated by the possibility that the DSN $70 \mathrm{~m}$ antennas could contribute to pulsar timing array measurements, particularly the $70 \mathrm{~m}$ antenna at the Canberra Deep Space Communications Complex (CDSCC), we undertook a project to design and deploy a new, wideband feed. Key requirements for the new feed was that it fit into the space occupied by the existing feed (both length and width), with no more than a modest increase in mass allowed.

The new feed is of a corrugated design, matched to a broadband polarizer and orthomode transducer (Hoppe et al. 2015). While a largely conventional design, considerable attention was paid to the mechanical design in order to avoid a large mass increase. Over the past 18 months, this new feed was designed, fabricated, and deployed to the Canberra $70 \mathrm{~m}$ antenna. Figure 1 shows the new feed being lifted and installed on the antenna.

Following installation (2015 August), we are characterizing the performance of the feed, and beginning the manufacture of the orthomode transducer to enable dual polarization observations.

\section{Acknowledgement}

The development and installation of this new feed would not have been possible without the support of many people at JPL and CDSCC. Part of this research was carried out at the Jet Propulsion Laboratory, California Institute of Technology, under a contract with the National Aeronautics and Space Administration. The NANOGrav Project receives support from NSF Physics Frontier Center award number 1430284. 


\section{References}

Backer, D. C., Kulkarni, S. R., Heiles, C., Davis, M. M., \& Goss, W. M. 1982, Nature, 300, 615

Bates, S. D., Lorimer, D. R., \& Verbiest, J. P. W. 2013, MNRAS, 431, 1352

Hellings, R. W. \& Downs, G. S. 1983, ApJ, 265, L39

Hoppe, D. J., Khayatian, B., Lopez, B., et al. 2015, Interplanetary Network Progress Report 42-202; http://http://ipnpr.jpl.nasa.gov/progress_report/42-202/202C.pdf

Lazio, J., Teitelbaum, L., Franco, M. M., et al. 2015, IAU XXIX General Assembly, \#2251715

Withington, J. 1988, Telecommunications \& Data Acquisition Progress Report, TDA PR 42-94; http://ipnpr.jpl.nasa.gov/progress_report/42-94/94X.PDF 